\title{
PROJECT VARIANT: CURRENT AND FIELD MEASUREMENTS ON BOARD SICH-1M SATELLITE
}

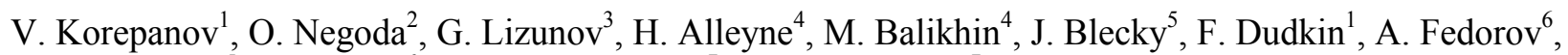 \\ J. Juchniewicz ${ }^{5}$, S. Klimov ${ }^{6}$, V. Krasnoselskikh ${ }^{7}$, and F. Lefeuvre ${ }^{7}$
}

${ }^{1}$ Lviv Centre of Institute of Space Researches (LCISR), 5-A Naukova str., 290601, Lviv, Ukraine

${ }^{2}$ National Space Agency of Ukraine, 11 Bozhenka str., 252022, Kyiv, Ukraine

${ }^{3}$ Kyiv University, 6 Glushkova str., 252022, Kyiv, Ukraine

${ }^{4}$ Sheffield University, Amy Johnson Building, Mappin Street, Sheffield, S1 3JD, United Kingdom

${ }^{5}$ Space Research Centre, 18-A Bartycka str., 00716, Warsaw, Poland

${ }^{6}$ Space Research Institute (IKI), 84/32 Profsoyuznaya str., 117810, Moscow, Russia

${ }^{7}$ LPCE/CNRS, 3A Avenue de la Recherche Scientifique, 45071, Cedex 02, Orleans, France

\begin{abstract}
VARIANT is a joint international space experiment on current density measurements in ionosphericmagnetospheric plasmas. The experiment will be performed onboard the Ukrainian remote sensing satellite SICH$1 \mathrm{M}$, that will be launched in 2000 at the polar circular orbit with the inclination of around $83^{\circ}$ and altitude $670 \pm 30$ $\mathrm{km}$. The scientific payload includes three instruments for registration of space current density: a split Langmuir probe, a Rogovski coil and a Faraday cup. The first two of these instruments are dedicated to measure current density variations and the last the particles' fluxes. The equipment also includes sensors for measurements of the electric and magnetic field fluctuations in the frequency range from $0.1 \mathrm{~Hz}$ to $40 \mathrm{kHz}$. Main objectives of the VARIANT mission are as follows:

- direct comparison of the spectral characteristics of the electric and magnetic fields with the characteristics of the field aligned currents in the polar regions; mapping of the field aligned current distribution;

- comparative study of the field aligned current structures with the characteristics of the ionospheric convection observed by the system of radars SuperDARN;

- comparative study of technological problems associated with different techniques of current density measurements;

and the secondary objectives are:

- active experiments with the onboard radar;

- registration of the signatures of the seismo-active and volcanic phenomena; investigation of the man-made impact upon the ionosphere (anthropogenic hazards, pollution, etc).
\end{abstract}

\section{INTRODUCTION}

It is well known that the parameters of the ionospheric plasma are primarily controlled by the solar activity. Their sporadic perturbations usually appear as a response to perturbations in the solar wind and magnetosphere. The problem of interaction between the solar wind, magnetosphere and ionosphere is one of the key problems in SolarTerrestrial Physics, and the wide scientific community works in special programs dedicated to it. Data base used for such studies includes data from ground based magnetic stations observations, ionospheric sounding and the of SuperDARN radars measurements of the magnetospheric convection phenomena and direct satellite 
measurements. The goal of these research programs is to develop the methods of ionospheric-magnetospheric monitoring and to be able to predict parameter variations.

The modeling of ionospheric-magnetospheric coupling is a very difficult task. First of all the system is connected to external fluxes of particles, energy and pulses. Second, the interaction process is rather complicated. The process of magnetospheric convection controlled by the solar wind is intrinsically unstable due to the presence of the conductive ionospheric layer. The ionosphere is not a passive "target" that absorbs wave and particle flows penetrating from the magnetosphere or "resistance" that short-circuits magnetospheric currents. It provides an active feedback impact on the perturbations of magnetosphere, and moreover can itself be the source of the unstable perturbations that in their turn can penetrate and disturb the magnetosphere. This interaction takes place by means of field aligned currents in the magnetosphere that are closed by ionospheric plasma through the transverse (Pedersen's and Hall's) conductivity. The variations of this conductivity (that can be caused by different factors such as, for instance, the ion drag process by the atmospheric winds or by inhomogeneous heating of the neutral gas or by some other factors) can modify current distribution and associated global structure of electric fields in the magnetosphere.

Even though there were a large number of satellite investigations of these phenomena (Bulgaria 1300, S33, GEOTAIL, FAST, INTERBALL, etc.) (Chmyrev et al, 1988; Heelis, 1988; Lysak and Carlson, 1982; Lysak and Dum, 1983; Titova et al, 1984), still there are many unanswered questions in the problem of ionospheremagnetosphere coupling. Namely, one can mention the following :

- electric current distribution at a day side of auroral ionosphere, its dependence on the interplanetary magnetic field orientation;

- electrodynamics of the polar cusp where plasma is strongly disturbed by interaction with solar wind;

- localization and structure of plasma convection cells of different spatial scales in the ionosphere (also dependent on interplanetary magnetic field parameters);

- statistical properties and analysis of the role of field aligned current structures in ionospheric-magnetospheric coupling.

Solar activity is the main factor that affects the ionosphere; another is the particles and energy flows penetrating up the ionosphere, from the Earth (Morena and Kazimirovsky, 1996). Recent observations reveal that the ionosphere locally reflects tropospheric phenomena (storms, upward shooting lightning, large cyclones and atmospheric fronts), anthropogenic factors (rocket launches, especially powerful ones, descent of spacecrafts, explosions, radio transmitter operations, etc.) and lithospheric processes (seismic activity, earthquakes, active fractures). The possibility to use these data for the prediction of earthquakes and monitoring of the anthropogenic and natural hazards is a new area of current research.

The observed seismo-activity influence on the ionosphere (Parrot, 1995) is rather weak and is usually masked by the solar activity effects. The seismogenic disturbances are typically detected in the ionosphere under low solar activity conditions $(\mathrm{Kp}<4)$. Nevertheless, making use of modern signal processing methods there is a hope to extract them if the structural peculiarities of seismogenic "signals" will be reliably determined. This approach, however, requires a huge amount of experimental data as well as appropriate theoretical modeling.

The major goal of the VARIANT project is to carry out direct measurements of current density in the ionosphere combined with the measurements of the magnetic and electric field fluctuations that can serve as new elements in the data set of signatures of the solar wind-magnetosphere-ionosphere coupling. The secondary goal of VARIANT experiment is the statistical study of minor seismogenic effects and their comparison with the "background noise statistical data set".

\section{PAYLOAD INSTRUMENTATION}

Following the major project goal - the study of the ionospheric plasma response on the influence of the magnetosphere and solar wind from the top side, and the atmosphere and the Earth, from the botom side, - a set of electromagnetic experiments onboard the Ukrainian remote sensing satellite $\mathrm{SICH}-1 \mathrm{M}$ is planned. The satellite 
will be launched in 2000 in a circular orbit with the altitude of $670 \pm 30 \mathrm{~km}$ and inclination angle of about $83^{\circ}$. It should cross the main morphological structures of the ionosphere: middle latitude throat, polar cusps, auroral oval.

Onboard satellite instrumentation includes three instruments for the registration of electric current density as the main signature of ionosphere-magnetosphere coupling. These particular instruments should allow in future the separation of the spatial and temporal variations onboard single satellite. This possibility is based on the idea to carry out the direct measurements of the current layers by crossing them (Vaisberg et al, 1989). This gives rise to the possibility to determine the current density of the plane wave, that really allows the independent evaluation of curl $\mathbf{B}$. If the magnetic field fluctuations are measured simultaneously one can get the distribution of the $\mathbf{k}$-vectors, and then calculate the real wave frequency (Krasnossel'skikh et al, 1991). The scientific equipment devoted to this task includes split Langmuir probe (WZ) (Vaisberg et al, 1989), Rogovsky coil (ZF) (Krasnossel'skikh et al, 1996) and Faraday cup (FC) (Safrankova et al, 1995). The simultaneous measurements of electric and magnetic field fluctuations in the ELF/VLF frequency band will be performed making use of the electric and magnetic fields sensors (instruments EZ and WZ). The waves in this frequency range are supposed to be associated with different types of the ionospheric disturbances. They are also assumed to be the most typical signatures of the seismogenic effects. Taking all this into account, the proposed payload includes 4 types of sensors (table 1).

Table 1. Scientific Payload Proposed for SICH-1M Mission

\begin{tabular}{|c|c|c|c|}
\hline 1 & Device & Measurement & Designed by \\
\hline 1. & Wave probe WZ & $\begin{array}{l}\text { Electric current density } \mathbf{J}: \\
\text { Frequency range } 0.1 \mathrm{~Hz} \ldots 40 \mathrm{kHz} \text {, } \\
\text { Noise } 10^{-12} \mathrm{~A} / \mathrm{cm}^{2} \mathrm{~Hz}^{1 / 2} \\
\text { Magnetic field vector } \mathbf{B} \text { : } \\
\text { Frequency range } 0.1 \mathrm{~Hz} \ldots 40 \mathrm{kHz} \\
\text { Noise } 10^{-13} \mathrm{~T} / \mathrm{Hz}^{1 / 2} \\
\text { Electric potential } \varphi \text { : } \\
\text { Frequency range } 0.1 \mathrm{~Hz} \ldots 40 \mathrm{kHz} \\
\text { Noise } 10^{-6} \mathrm{~V} / \mathrm{Hz}^{1 / 2}\end{array}$ & $\begin{array}{l}\text { LC ISR, Ukraine } \\
\text { (V. Korepanov) } \\
\text { IKI, Russia } \\
\text { Klimov) } \\
\text { CBK, Poland } \\
\text { (J. Juchniewicz) }\end{array}$ \\
\hline 2. & Rogovsky coil ZF & $\begin{array}{l}\text { Electric current density } \mathbf{J}: \\
\text { Frequency range } 0.1 \mathrm{~Hz} \ldots 400 \mathrm{~Hz} \text {, } \\
\text { Noise } 10^{-12} \mathrm{~A} / \mathrm{cm}^{2} \mathrm{~Hz}^{1 / 2}\end{array}$ & $\begin{array}{l}\text { LPCE/CNRS, France } \\
\text { (V. Krasnosselskikh) }\end{array}$ \\
\hline 3. & Electric probe EZ & $\begin{array}{l}\text { Electric field vector } \mathbf{E}: \\
\text { Frequency range } 0.1 \mathrm{~Hz} \ldots 200 \mathrm{kHz} \\
\text { Noise } 10^{-6} \mathrm{~V} / \mathrm{Hz}^{1 / 2} \\
\end{array}$ & $\begin{array}{l}\text { LC ISR, Ukraine } \\
\text { (V. Korepanov) }\end{array}$ \\
\hline 4. & Faraday cup FC & $\begin{array}{l}\text { Electric current density } \mathbf{J}: \\
\text { Frequency range } 0.1 \mathrm{~Hz} \ldots 1 \mathrm{kHz}, \\
\text { Noise } 10^{-10} \mathrm{~A} / \mathrm{cm}^{2} \mathrm{~Hz}^{1 / 2}\end{array}$ & $\begin{array}{l}\text { Sheffield University, United } \\
\text { Kingdom } \\
\text { (H. Alleyne, M. Balikhin) }\end{array}$ \\
\hline 5. & DC magnetometer FZM & $\begin{array}{l}\text { Magnetic field vector } \mathbf{B} \\
\text { Frequency range DC }-1 \mathrm{~Hz}\end{array}$ & $\begin{array}{l}\text { LC ISR, Ukraine } \\
\text { (R. Berkman, S. Belyayev) }\end{array}$ \\
\hline
\end{tabular}

The spectral noise density of all sensors is given for the frequency $100 \mathrm{~Hz}$. The spatial density of electric current will be simultaneously measured by three independent instruments: split Langmuir probe (included into the wave probe unit WZ), Rogovsky coil (ZF) and Faraday cup (FC). These measurements represent a special and very important task of the experiment which procures the unique possibility to justify, validate and compare three existing methodologies of the ionospheric current registration in space experiment as well as to perform the intercalibration of the instruments. The proposed scientific payload also includes a VLF analyser and allows fulfilment particular objectives of SICH-1M experiments.

\section{SCIENTIFIC GOALS OF VARIANT PROJECT}

Investigation of the Global Distribution of Field Aligned Currents, Large Scale Electric Fields and Ionospheric Plasma Convection 
Combined investigation of the high latitude ionosphere reveals the mechanisms of energy transfer through the chain 'solar wind $\rightarrow$ magnetosphere $\rightarrow$ ionosphere'. Charged particle beams ejected from the magnetotail to ionosphere precipitate in aurorar oval zones. Solar wind particles can directly penetrate into ionosphere through the polar cusp causing optical emissions and additional plasma ionisation. Due to the high transverse conductivity (Hall and Pedersen) longitudinal currents circuit by ionosphere leads to the modification of electric field distribution in the magnetosphere. Finally, the global distribution of electric currents reflects the structure of magnetospheric fields as well as the ionospheric plasma state (inhomogeneity of the transverse ionospheric conductivity, peculiarities in neutral atmosphere motion, etc.) (Chmyrev et al, 1988; Feldstein and Galperin, 1985; Heelis, 1988; Heppner, 1972; Lysak and Carlson, 1981; Lysak and Dum, 1983; Titova et al, 1984). Experimental investigation of these phenomena requires the measurement of currents and electric and magnetic fields in the high latitude ionosphere what is the major goal of the VARIANT mission.

Correlated Study of the Field Aligned Current Variations and the Small Scale Structure of the Magnetospheric Convection Making Use of Simultaneous Measurements of the Radar Chain SuperDARN and SICH-1M Satellite

SuperDARN radar network is dedicated to study the magnetospheric convection in polar regions by means of incoherent scattering. The northern hemisphere network currently consists of six radars that determine two components of bulk velocity of plasma in the E- and F-regions. The measurements are provided in the regime of monitoring and the distribution of data in the form of a CD-set is available. LPCE group (J.-P. Villain and R. Andre) carried out the study of the phenomenon of the demagnetization of ions and the formation of the small scale divergent structure during the process of the reconstruction of the convection pattern in the evening sector. It is supposed that this process is accompanied by the field aligned currents in the magnetosphere and ionosphere (Oliver et al, 1983). We propose to carry out simultaneous measurements using ground based radar facilities and VARIANT onboard measurements.

Investigation of Wave Processes in Polar Cusp Plasma

The proposed scientific payload allows one to measure the electromagnetic field and electric current fluctuations in the frequency range that contains characteristic plasma emissions associated with lower hybrid oscillations, ion cyclotron waves and ion acoustic waves, as well as wide band emissions at the ionospheric level. These emissions are characteristic for the polar cusp region which is well covered by the proposed SICH-1M satellite orbit.

The polar cusp is a region of free and direct access of the solar wind into the inner magnetosphere and it is assumed also to be the site of the turbulent plasma flow. Theoretical models of the solar wind interaction with the Earth's magnetosphere predict the presence of complicated structures of the plasma flow in the cusp's narrow neck with vortices and turbulent zones of field line reconnection. At lower altitudes strong field aligned current sheets and filaments accompanied by ion cyclotron and higher frequency waves have been discovered by the OGO-5 and VIKING satellites (Woch and Lundin, 1992). The investigation of plasma wave spectra in the region of the polar cusp is the one of the goals of proposed experiment.

\section{Study of the ELF/VLF Emissions in the Ionosphere Associated with the Seismic Activity}

Small variations of the ionospheric plasma parameters can carry indications of the Earth's seismic activity. In a few hours or days before earthquakes the variations of spectra and intensity of air glow, ULF-VLF electromagnetic fields, various ionospheric plasma phenomena are detected by satellites and ground based facilities in the vicinity of the epicentre of the future earthquake. Today the understanding of the nature of these phenomena is far from being complete. Its investigation is related to such problems of ionospheric plasma physics as energetic balance of the ionosphere and its properties as the electromagnetic medium.

Electromagnetic emissions are the typical signatures of seismogenic effects in the ionosphere (Parrot, 1995). Most of the observations onboard satellites that have detected such emissions observed in the VLF range (as whistler mode) and more seldom in the ULF range (as Alfven mode). A great amount of data have been obtained by INTERCOSMOS-18,19 and 24, OGO-6, NIMBUS, AUREOL-3, GEOS-2, INTERCOSMOS-BULGARIA-1300, DE-2, COSMOS-1809 missions. Specific noise-like VLF electrostatic emissions have been detected in the range of 
few tens $\mathrm{kHz}$ over the earthquake epicentre within 200 to $300 \mathrm{~km}$ in the latitudinal direction and over a greater distance in the longitudinal direction. These emissions originate about 10 to 20 hours before the main shock and continue over about the same time after it. They reach their intensity peak at the instant of the main shock. ULF $\sim 1$ Hz-oscillations have been recorded several hours before the main shock in a narrow magnetic field tube (40...100 $\mathrm{km}$ along the satellite trajectory) originating in the epicentre of the earthquake. In the case of moderate earthquake $(\mathrm{M}=3.5 \ldots 5)$ the magnetic field disturbances have values of the order of $0.2 \ldots 0.5 \mathrm{nT} / \mathrm{Hz}^{1 / 2}$ at altitudes of 800 to 900 $\mathrm{km}$ and frequency about $8 \mathrm{~Hz}$. To improve the reliability of such data is also one of the goals of this proposal.

Investigation of the Infrasonic Wave Effect upon the Ionosphere; Active Experiments with Ground Based Sources of Powerful Acoustic Emission

Physical mechanisms of seismic effect upon the ionosphere are not well established. One hypothesis is that the ionosphere experiences the influence of atmospheric acoustic gravity waves emitted by seismic oscillations of the Earth surface. Since the frequencies of seismic waves range in the band $0.001 \ldots 1 \mathrm{~Hz}$ it is natural to speak in terms of the infrasonic channel of lithosphere-ionosphere coupling. It is supposed that the dissipation of acoustic gravity waves may trigger the instability of the magnetospheric convection and give rise to the development of perturbations, that can in their turn propagate from the ionosphere to upwards in the form of the Alfven waves. It can be assumed that the crossing of the ionospheric dynamo region (at height $80 \ldots 150 \mathrm{~km}$ ) by the infrasonic waves can generate electromagnetic Alfven waves with the same frequency. Such waves may be registered onboard the satellite.

LCISR operates powerful ground based sources of acoustic radiation. Preliminary experiments demonstrate the effect of acoustic waves on the ionosphere including the generation of ELF magnetic field perturbations caused, probably, by Alfven waves generation. The SICH-1M mission provides a good opportunity to carry out combined experiments with the ground based emission of acoustic waves and the detection of ionospheric plasma response by the satellite.

\section{Modification of the Satellite Plasma Environment by the Operation of Onboard Radar}

The VARIANT experiment is a secondary one onboard remote sensing SICH-1M satellite. The SICH-1M principal payload contains a powerful radar for Earth observation. The modification of the plasma environment as a result of the active operation of this radar is one of the important problems which also can be studied. Previous studies reveal that the operation of a powerful (onboard) transmitter leads to the excitation of a number of plasma resonance in satellite proximity. The unique combination of three instruments for the registration of plasma current and the electromagnetic field in VARIANT experiment will allow us for the first time to study experimentally the wave vectors which corresponds to different resonances and to determine experimentally the waves dispersion in plasma reference frame.

\section{GROUND SUPPORT}

One of the main goals of the VARIANT experiment is the comparison between different measurement methods. It supposes that each instrument will be thoroughfully tested separately first. Then all experiments will be integrated and their joint operation investigated with the aim to study the radiation of all sub-systems, both of the scientific payload and of the satellite. Also ground tests involving data simulation and decomposition of output files and their comparison are planned.

The ground support program has to involve the development of procedures for data collection, transmission and storage. Also a data evaluation program has to be developed for quick-look information, test of satellite operation, calibration and finally data validation and archiving.

It is necessary to foresee during the active phase of the experiment the possibility of changing the on-board software both for the satellite subsystems and scientific payload, in order to develop the procedure of telecommands and telemetric data acquisition. Taking into account the originality of the project and the 
importance of obtained information for further interpretation, data validation and archiving procedure has to be developed. Collaboration with the "Centre Francais des Donnees de la Physique de Plasmas" (CDPP) is planned. A special program will be arranged to determine the data format with the requirements of CDPP.

\section{ACKNOWLEDGEMENTS}

This work has been partially supported by NSAU Contract № 1189, INTAS grant 97-1769 and RFFI grant 98-0217426.

\section{REFERENCES}

Chmyrev, V.M., S. Bilichenko, O. Pokhotelov, V. Marchenko, V. Lazarev, A. Streltsov, L. Stenflo, Alfven Vortices and Related Phenomena in the Ionosphere and Magnetosphere, Physica Scripta, 38, 841 (1988).

Feldstein, Ya.I., and Yu.I. Galperin, The auroral luminosity structure in the high-altitude upper atmosphere: its dynamics and relation to the large-scale structure of the Earth magnetosphere, Rev. Geophys., 23, pp. 217-275 (1985).

Heelis, R.A., Studies of ionopsheric plasma and electrodynamics and their application to ionospheremagnetosphere coupling, Rev. Of Geophysics., 26, No 2, pp. 317-328, (1988).

Heppner, J.P., Polar cap electric field distribution related to the interplanetary magnetic field direction, J. Geophys. Res., 77, 4877, (1972).

Krasnosel'skikh, V., A. M. Natanzon, A. E. Reznikov et al, Current Measurements in Space Plasmas and the Problem of Separating between Spatial and Temporal Variations in the Field of a Plane Electromagnetic Wave, Advances in Space Research, 11, N9, pp. 37-40 (1991).

Krassnosel'skikh, V., O. Randriamboarison et al, Floating potential of the space station and current density measurements around it, ESA (SP-385, December 1996), p. 347-351.

Lysak, R.L., and C.W. Carlson, The effect of microscopic turbulence on magnetosphere-ionosphere coupling, Geophys. Res. Lett., 8, 269 (1981).

Lysak, R.L., and S.T. Dum, Dynamics of magnetosphere-ionosphere coupling including turbulent transport, $J$. Geophys. Res., 88, 365 (1983).

Morena, B. and E. Kazimirovsky, The experimental investigation of the lower ionosphere responce to forcing from above and below, Sci. Reports. INTA 1995, El Arenosillo, (1996).

Oliver, W.L., J.M. Holt, R.H. Wand, and J.V. Evans, Millstone Hill incoherent scatter observation of auroral convection over $60^{\circ}<\Lambda<75^{\circ} 3$. Average patterns versus Kp, J. Geophys. Res., 88, 5505, (1983).

Parrot M., Electromagnetic Noise Due to Earthquakes, in Handbook of Atmospheric Electrodynamics, II, p. 95116, CRC Press, 1995.

Safrankova, J., G. Zastenker et al., Omnidirectional plasma sensor VDP, Interball. Mission and payload, CNESIKI (1995), p. 195-198.

Titova, V., E. Yurov, O. Molchanov, V. Krasnosel'skikh, et al, Small Scale Structures of Electric Field Variations and Particle Precipitations as Observed onboard of Aureol-3 Satellite, in Proceedings of "Results of the ARCAD-3 Project and of the Recent Programs in Magnetospheric and Ionospheric Physics"; p. 447, CEPADUES-EDITION, France (May, 1984).

Vaisberg, O., On the Determination of the Spatial Scale in the Moving Reference Frame, Soviet Journal Space Research, 12, $\mathrm{N}^{\circ} 6$ (1985).

Vaisberg, O., S. Klimov, and V. Korepanov, Current Density Measurements in the Shock Front by means of Splitted Langmuir Probe, Soviet Journal Space Research, 27, № 3 (1989).

Woch, J., and R. Lundin, Signatures of transient boundary layer processes observed by VIKING, J. Geophys. Res., 97, 1431-1447, (1992). 